\title{
Constituent-quark model description of triply heavy-baryon nonperturbative lattice QCD data
}

\author{
J. Vijande \\ Departamento de Física Atómica, Molecular y Nuclear, \\ Universidad de Valencia (UV) and IFIC (UV-CSIC), E-46100 Valencia, Spain \\ A. Valcarce \\ Departamento de Física Fundamental and IUFFyM, \\ Universidad de Salamanca, E-37008 Salamanca, Spain \\ H. Garcilazo \\ Escuela Superior de Física y Matemáticas, \\ Instituto Politécnico Nacional, Edificio 9, 07738 México D.F., Mexico
}

(Dated: Version of August 20, 2018)

\begin{abstract}
This paper provides results for the spectra of triply charmed and bottom baryons based on a constituent quark model approach. We take advantage of the assumption that potential models are expected to describe triply heavy baryons to a similar degree of accuracy as the successful results obtained in the charmonium and bottomonium sectors. The high precision calculation of the ground state and positive and negative parity excited states recently reported by nonperturbative lattice QCD provides us with a unique opportunity to confront model predictions with data. This comparison may also help to build a bridge between two difficult to reconcile lattice QCD results, namely, the lattice SU(3) QCD static three-quark potential and the recent results of nonperturbative lattice QCD for the triply heavy-baryon spectra.
\end{abstract}

PACS numbers: 14.40.Lb,12.39.Pn,12.40.-y 


\section{INTRODUCTION}

In a previous publication [1] we have pointed out that the static three-quark potential with parameters determined from SU(3) lattice QCD [2] does not reproduce the triply heavybaryon $b b b$ and $c c c$ spectra measured also in lattice QCD [3 5]. We argued several possible reasons for such disagreement. In this work we aim to analyze whether the triply heavybaryon spectra recently calculated by means of nonperturbative lattice QCD techniques can be understood within a constituent quark-model framework by means of simple Cornell-like potentials as it is the case of charmonium and bottomonium spectra [6 8]. This calculation may help to connect the description of the heavy-baryon spectra obtained by nonperturbative lattice QCD techniques and the static potentials derived within SU(3) lattice QCD.

Theoretically, one would expect that potential models would be able to describe triply heavy baryons to a similar degree of precision as their success in charmonium and bottomonium. As noticed by Bjorken some time ago [9], bound states of three-heavy quarks reveal a pure baryonic spectrum without light-quark complications. In the same way the $Q \bar{Q}$ interactions are examined in heavy mesons, the study of triply heavy baryons will probe the $Q Q$ interactions in the heavy quark sector. However, no experimental results are available so far for triply heavy baryons (see Ref. [10] for a recent calculation of production cross section at the LHC), and thus, the predictions of their properties cannot yet be compared to the real world. The recent precise calculation of the ground and excited states of triply bottom

baryons [3, 4], together with the ground and excited states of triply charm baryons [5], provides us with a unique opportunity to test phenomenological quark models for baryons in the energy regime in which the description using potential models is expected to work best, the heavy-quark sector. Hence, the quark-model dependent calculations could be tested by comparing them to nonperturbative first-principles calculations in lattice QCD of the $b b b$ and $c c c$ systems.

The paper is organized as follows. In the next section we will briefly described the Cornell potential used for the description of the heavy-meson sector. We will try to connect the well established parameters used in the literature for the heavy-meson sector into the relatively unknown heavy-baryon sector. In Sec. III we will present our results. We will firstly analyze the pattern of the nonperturbative lattice QCD results looking for the general structure of the potential. We will pursue different parametrizations in a trial to get a unified description of the $b b b$ and $c c c$ spectra. The results will be derived by different numerical techniques previously tested by our group: generalized Gaussians variational approaches, hyperspherical harmonics and Faddeev equations [11 13]. Finally, in Sec. IV, we will summarize the main conclusions of this study.

\section{A CONSTITUENT QUARK MODEL POTENTIAL FOR THREE-HEAVY QUARKS.}

Since the early days of QCD the interaction among heavy quarks has been explored as an important tool to learn about the behavior of QCD at low energies. At the end of 1974, when the new particles seen at Brookhaven and SLAC were identified as $c \bar{c}$ bound states, explicit models were proposed to calculate the spectrum and the radiative transitions (see Ref. [14] for a recent pedagogical review). The potential proposed in [15] is known as the 
funnel or Cornell potential and it reads,

$$
V_{i j}^{Q \bar{Q}}(r)=-\frac{a}{\left|\vec{r}_{i}-\vec{r}_{j}\right|}+b\left|\vec{r}_{i}-\vec{r}_{j}\right|+c .
$$

Solving the two-body problem, one can tune the parameters to reproduce the low levels of charmonium. This was done by several groups in the 70's and the authors were able to predict the missing states. The game became more challenging when the first bottomonium levels were found, trying to reproduce simultaneously the $c \bar{c}$ and $b \bar{b}$ spectra. Indeed, even so the interquark potential was not derived from QCD in early quarkonium phenomenology, it was assumed to be universal, or flavor independent. In QCD the gluons couple to the color, hence it is reasonable to assume that the potential is flavor independent.

The $Q \bar{Q}$ potential has also been extensively studied by lattice gauge theories [16], being nowadays a very well-known quantity resembling the structure derived from the heavy-meson spectra. Thus, the typical shape of the color-singlet $Q \bar{Q}$ potential is characterized by a shortrange Coulomb behavior and a long-range linear rise, that well represents the double nature of QCD as an asymptotically free and infrared confined theory. The excitation spectrum of the gluon field around a static quark-antiquark pair has also been explored by lattice calculations [17]. On the large length scale the spectrum agrees with that expected for string-like excitations while in the short range it shows a Coulomb-like behavior as it was first noted within the context of the static bag picture of gluon excitations [18].

The $3 Q$ potential should be the analog of the famous Cornell potential for quarkonium. The short-distance behavior of $V^{3 Q}(r)$ is expected to be described by the two-body Coulomb potential as the one-gluon exchange result in perturbative QCD. It should be extended for the baryon case, with a factor $1 / 2$ in front of its strength due to color factors [14]. As for the $Q \bar{Q}$ case, the characteristic signature of the long-range non-Abelian dynamics is believed to be a linear rising of the static interaction. Moreover, the general expectation for the baryonic case is that, at least classically, the strings meet at the so-called Fermat (or Torricelli) point, which has minimum distance from the three sources ( $Y$-shape configuration) [19, 20]. The confining short-range $3 Q$ potential could be also described as the sum of two-body potentials ( $\Delta$-shape or linear configuration) [19 22]. We have demonstrated in Ref. [1] the equivalence of both prescriptions for the case of triply heavy baryons (see Table II of that reference) and for different values of the heavy-quark mass. Thus, a minimal model to study $3 Q$ systems may come given by,

$$
V^{3 Q}(r)=-A \sum_{i<j} \frac{1}{\left|\vec{r}_{i}-\vec{r}_{j}\right|}+B \sum_{i<j}\left|\vec{r}_{i}-\vec{r}_{j}\right|+C .
$$

The value of the $Q \bar{Q}$ confinement strength $b$ is usually fixed to reproduce that obtained from the linear Regge trajectories of the pseudoscalar $\pi$ and $K$ mesons, $\sqrt{\sigma}=(429 \pm 2)$ $\mathrm{MeV}$ [16]. In the case of baryons, the linear string tension $B$ is considered to be of the order of a factor $1 / 2$ of the $Q \bar{Q}$ case. The reduction factor in the string tension can be naturally understood as a geometrical factor rather than a color factor, due to the ratio between the minimal distance joining three heavy quarks and the perimeter length of a $3 Q$ triangle, suggesting $B=(0.50 \sim 0.58) b[2]$. For the particular case of quarks in an equilateral triangle $B=\frac{1}{\sqrt{3}} b=0.58 b[20]$. When the linear ansatz is adopted for the two-body potential, still the same relation holds for the strength of the Coulomb potential $A \simeq \frac{1}{2} a$, due to color factors. The $\Delta$ ansatz (linear potential) has been widely adopted in the nonrelativistic quark model because of its simplicity [12, 13, 23, 27] 


\section{RESULTS AND DISCUSSION}

To check whether the Cornell-like potential of Eq. (21) reproduces the $b b b$ and $c c c$ baryon spectra measured in lattice QCD [3 -5] we will make use of three different numerical methods: the generalized Gaussians variational approach [11], hyperspherical harmonics [12] and Faddeev equations [13]. The three methods have been used and the difference in results is negligible. In all cases we solve the nonrelativistic Schrödinger equation

$$
\left\{H_{0}+V^{3 Q}(r)\right\} \Psi(\vec{r})=E \Psi(\vec{r}),
$$

where $H_{0}$ is the free part of three-heavy quarks without center-of-mass-motion

$$
H_{0}=\sum_{i=1}^{3}\left(M_{Q}+\frac{\vec{p}_{i}^{2}}{2 M_{Q}}\right)-T_{C M}
$$

and $M_{Q}$ is the mass of the heavy quark. The mass of the heavy baryon will be finally given by $M_{3 Q}=3 M_{Q}+E$.

It has been demonstrated in Ref. [1] that the results of nonperturbative lattice QCD for the $b b b$ and $c c c$ systems are not reproduced by the static three-quark potential derived in SU(3) lattice QCD [2]. We had hypothesized on several possible reasons for this disagreement. Among them, we suggested the possibility that the parameters obtained in quenched QCD in Ref. [2] might be different from those in 2+1 flavor QCD employed in Refs. [3 [5]. To check this possibility parameters in $2+1$ flavor QCD has to be obtained, unfortunately such calculations do not exist so far. We also wondered about the possibility that the fitting form Eq. (2) might not be appropriate to describe the static three-quark potential in lattice QCD or that the quark model description with "3-quark potential" might be inappropriate for $b b b$ and $c c c$ systems. We will try to mark out these possibilities by studying the nonperturbative lattice QCD data in terms of the suggested Cornell-like potential of Eq. (2) with parameters adjusted to the data, looking also for the possible need of higher order terms.

In Ref. [1] it has been noticed how $b b b$ nonperturbative lattice QCD calculations point to a $3 Q$ potential given by a mixture of a linear confinement and a Coulomb interaction. The comparison performed of the $b b b$ and $c c c$ nonperturbative lattice QCD results and calculations based on a mixture of a $\Delta$-shape confinement and a Coulomb interaction using the parameters reported in Ref. [2] $\left(A=0.1410\right.$ and $\left.B=0.0925 \mathrm{GeV}^{2}\right)$ showed a large difference in the excited states, predicting a small splitting between positive and negative parity excited states and also a small excitation energy for the positive parity states. These results may point to a lack of strength either in the confining or in the Coulomb potential.

We have analyzed which of the two terms, linear confinement or Coulomb, would play the relevant role by keeping the strength of one of them as predicted by SU(3) lattice QCD and increasing the other to pursue a spectrum close to the nonperturbative lattice QCD results of Refs. [3 5]. We show in Fig. 1] results for the $b b b$ system. In the upper panel we have kept constant the strength of the linear confining interaction, $B=0.0925 \mathrm{GeV}^{2}$, and we have augmented the strength of the Coulomb potential up to $A=0.2787$, a value rather close to the $Q \bar{Q}$ Coulomb strength [2]. Alternatively, in the lower panel we have kept constant the strength of the Coulomb potential $A=0.1410$, and we have increased the strength of the linear confining interaction up to $B=0.1517 \mathrm{GeV}^{2}$, which is also rather close to the $Q \bar{Q}$ strength [2]. One can easily identify the peculiarities of three identical heavy-quark baryons. By increasing the Coulomb potential, upper panel, the mass of all excitations is 

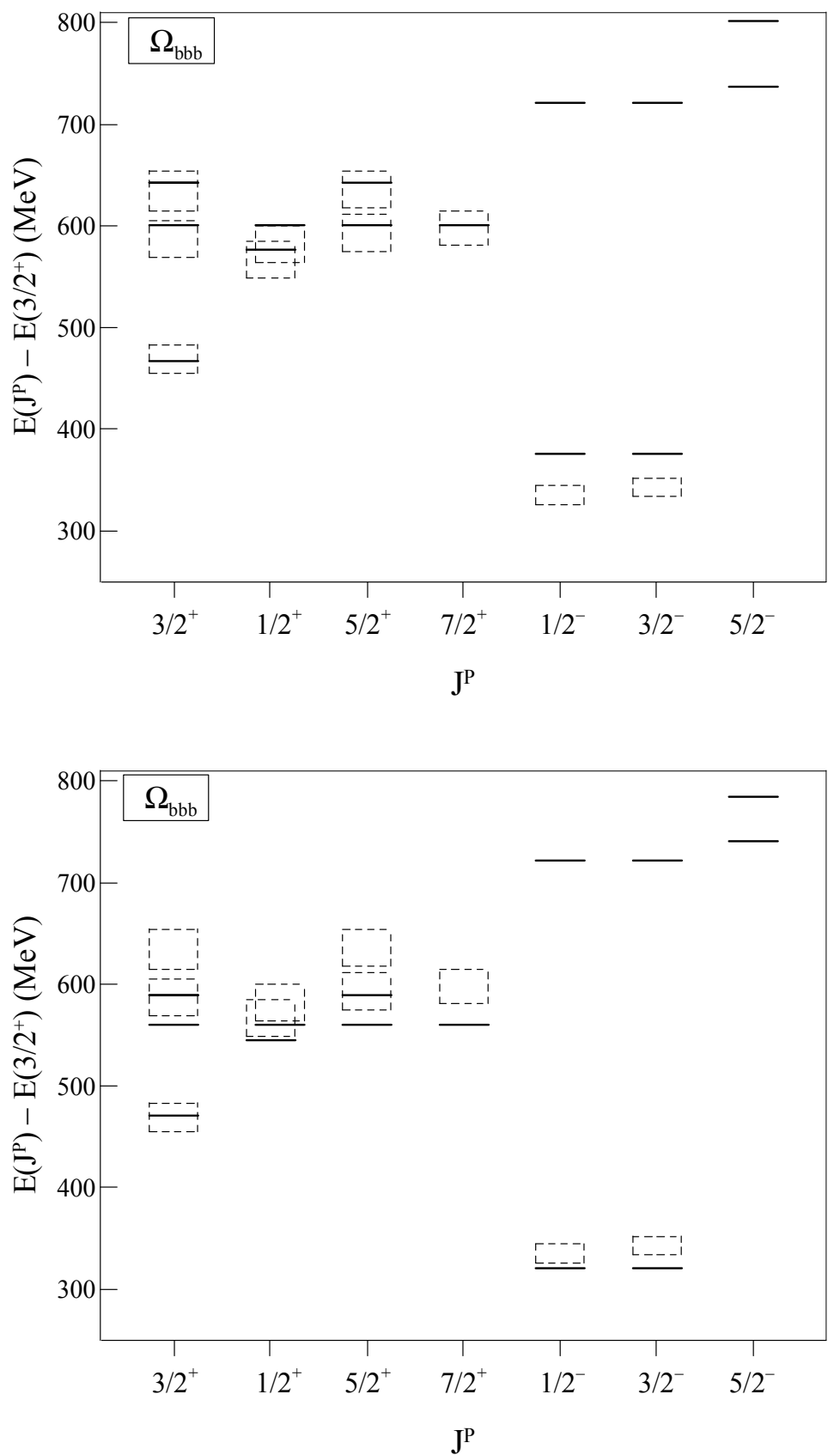

FIG. 1: $b b b$ excited state spectra, solid lines, for the potential of Eq. (2) with $A=0.2787$ and $B=$ $0.0925 \mathrm{GeV}^{2}$ (upper panel) or $B=0.1517 \mathrm{GeV}^{2}$ and $A=0.1410$ (lower panel). See text for details. The boxes stand for the nonperturbative lattice QCD results of Ref. [4].

increased but the mass difference between the positive and negative parity excited states, $E_{2}\left(3 / 2^{+}\right)-E_{1}\left(1 / 2^{-}\right)$, is smaller than predicted by nonperturbative lattice QCD. This mass difference could be enlarged by increasing the strength of the linear confining potential as we have done in the lower panel, but without lowering too much the mass of the negative parity states, $E_{1}\left(1 / 2^{-}\right)$and $E_{1}\left(3 / 2^{-}\right)$. It seems therefore that a moderate combined increase of the strength of the two terms, Coulomb and confinement, may allow to get a better fit to the nonperturbative lattice QCD data.

Thus, we have found a nice fit of the nonperturbative lattice QCD results with the poten- 

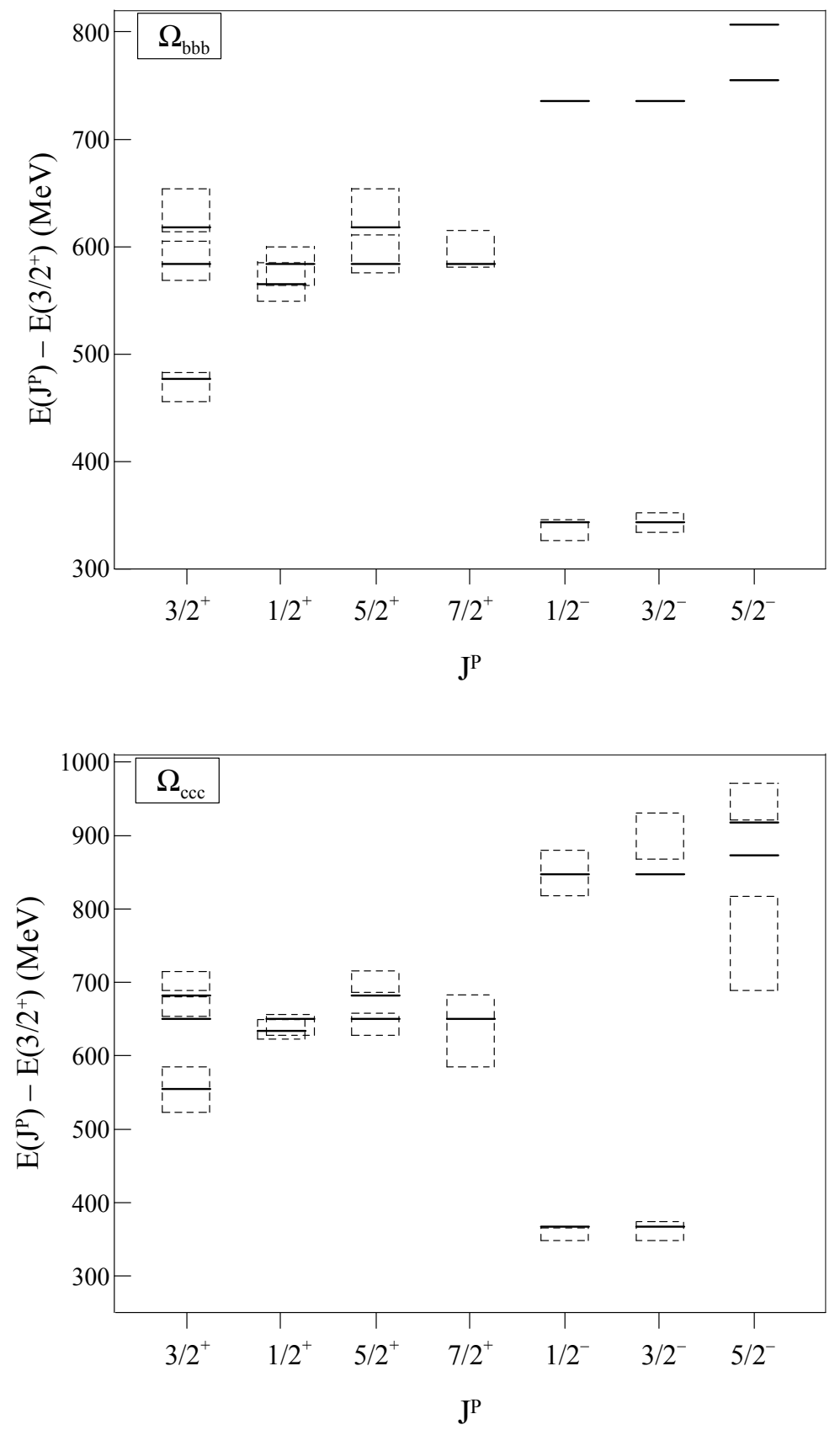

FIG. 2: $b b b$ (upper panel) and $c c c$ (lower panel) excited state spectra, solid lines, for the potential of Eq. (2) with the parameters $A=0.1875$ and $B=0.1374 \mathrm{GeV}^{2}$. The boxes stand for the nonperturbative lattice QCD results of Ref. [4] for the bbb system and Refs. [5, 28] for the $c c c$ system.

tial of Eq. (2) and the parameters $A=0.1875$ and $B=0.1374 \mathrm{GeV}^{2}$. The results are shown in Fig. 2 for the $b b b$ and $c c c$ systems. At this point one should bear in mind that the description of the charmonium and bottomonium experimental data with a Cornell-like potential needs of a large value of the $Q \bar{Q}$ Coulomb strength, $a \simeq 0.51-0.52[\underline{6}[\underline{8}]$, as compared to that obtained in SU(3) lattice QCD [2]. Using the phenomenological values reproducing the experimental data of the heavy quark meson spectra and the nonperturbative lattice QCD results for the triply heavy baryon spectra, one would conclude that $A / a<1 / 2$, slightly 
TABLE I: Excited states, in $\mathrm{MeV}$, of $c c c$ and $b b b$ systems from nonperturbative lattice QCD, Refs. [5, 28] and Ref. [4] and results for the $A L 1$ potential model of Ref. [25].

\begin{tabular}{|l|cc|cc|}
\hline & \multicolumn{2}{|c|}{$c c c$} & \multicolumn{2}{c|}{$b b b$} \\
& Ref. [25] & Refs. [5, 28] & Ref. [25] & Ref. [4] \\
\hline$E_{2}\left(\frac{3}{2}^{+}\right)-E_{1}\left(\frac{3}{2}^{+}\right)$ & 472 & $554 \pm 31$ & 451 & $469 \pm 14$ \\
$E_{3}\left(\frac{3}{2}^{+}\right)-E_{1}\left(\frac{3}{2}^{+}\right)$ & 567 & $667 \pm 13$ & 581 & $587 \pm 18$ \\
$E_{1}\left(\frac{1}{2}^{+}\right)-E_{1}\left(\frac{3}{2}^{+}\right)$ & 542 & $636 \pm 13$ & 555 & $567 \pm 18$ \\
$E_{1}\left(\frac{1}{2}^{-}\right)-E_{1}\left(\frac{3}{2}^{+}\right)$ & 316 & $357 \pm 9$ & 360 & $335.5 \pm 9.5$ \\
\hline
\end{tabular}

different from $1 / 2$ as the one-gluon exchange result. This conclusion could have been already anticipated from models designed to describe the light and strange baryon spectra and later extrapolated to triply-heavy baryons. The $A L 1$ model of Ref. [25] made use of $A=0.2534$ and $B=0.0827 \mathrm{GeV}^{2}$. We show in Table I the triply heavy baryon spectra obtained with this potential model as compared to the recent nonperturbative lattice QCD results. We observe how for the $b b b$ system, it drives to a too high negative parity states together with a small splitting between positive and negative parity states (a similar situation to the upper panel of Fig. 1) as a consequence of the large strength of the Coulomb potential. Besides, for the $c c c$ system this model drives to rather low excited states as a consequence of the small strength of confinement.

The description of the $b b b$ system shown in Fig. 2 has been obtained with a reasonable mass for the $b$ quark, $m_{b}=4.655 \mathrm{GeV}$, however in the case of the $c c c$ system one needs a large unrealistic mass for the $c$ quark, $m_{c}=2.050 \mathrm{GeV}$, what would drive to a $c c c$ ground state with a mass around $7 \mathrm{GeV}$. This seems to indicate that triply charm baryons present a more involved structure that does not fit so nicely in a simple pairwise linear plus Coulomb interaction. Such deviation may has partially its origin on the fact that the lattice QCD calculation of Ref. [5] did not address all the systematic uncertainties, but only statistical uncertainties are given. In fact, a calculation of the charmonium spectrum with the same lattice action and the same lattice spacing can be found in Ref. [29] where one can get an idea of the typical size of the systematic uncertainties. On the other hand, potential models probably are also less accurate for $c c c$ than for $b b b$ baryons, because the $c c c$ system is more relativistic and spin-dependent contributions may start playing a significant role. Although of small importance in heavy quark systems for being suppressed as $M_{Q}^{-2}$, the spin-spin interaction derived from the OGE may help to improve the description of the nonperturbative lattice QCD results. One may therefore add a spin-spin term to the interacting potential, having the final form,

$$
V_{S S}^{3 Q}(r)=-A \sum_{i<j} \frac{1}{\left|\vec{r}_{i}-\vec{r}_{j}\right|}+B \sum_{i<j}\left|\vec{r}_{i}-\vec{r}_{j}\right|+\frac{A}{M_{Q}^{2}} \frac{e^{-r / r_{0}}}{r r_{0}^{2}} \vec{\sigma}_{i} \cdot \vec{\sigma}_{j} .
$$

We have performed a fit to the $c c c$ data using the same Coulomb and linear confining strengths used for the $b b b$ system, $A=0.1875$ and $B=0.1374 \mathrm{GeV}^{2}$. When the spin-spin term is considered, one can reduce the value of the charm quark mass preserving a nice agreement with data. We show in Fig. 3 the results for standard values of quark-potential models, $m_{c}=1.6 \mathrm{GeV}$ and $r_{0}=0.28 \mathrm{fm}$ [25]. As one can see there is a good agreement, 


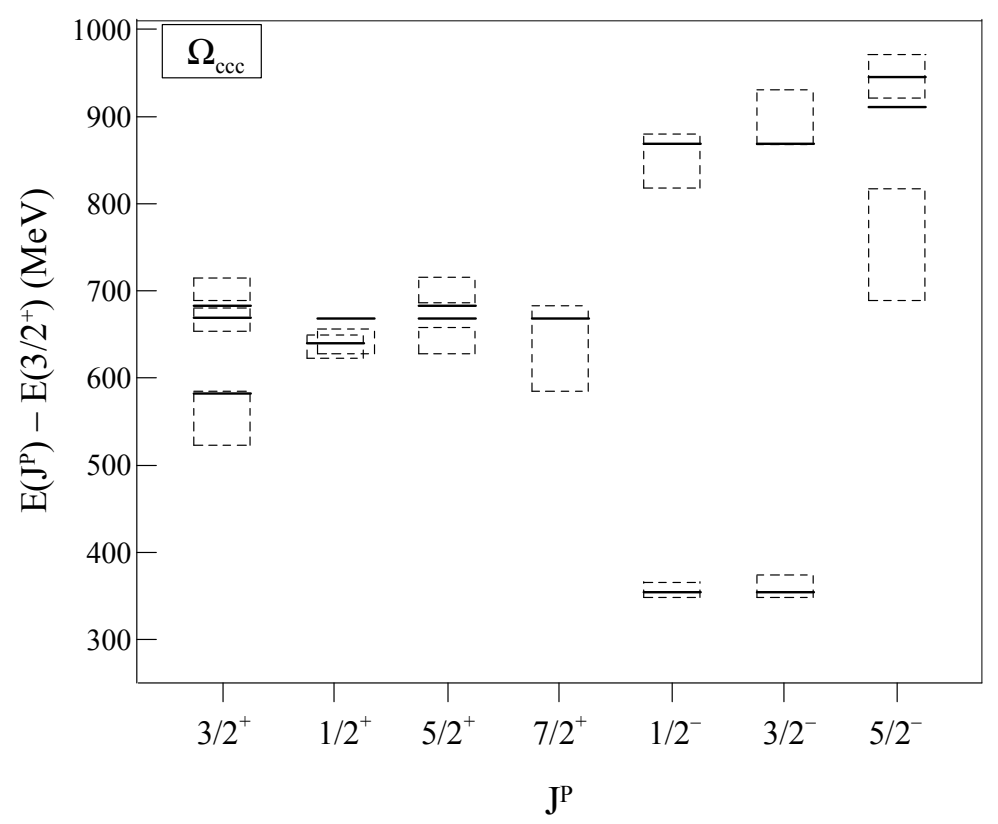

FIG. 3: ccc excited state spectra, solid lines, for the potential of Eq. (3) including a spin-spin interaction. The boxes stand for the results of Ref. [5].

sustaining the result of a short-range Coulomb potential supplemented by a linear confining interaction. The short-ranged spin-spin interaction helps to the fine tuning of the negative parity excited states (where it is negligible) with respect to the positive parity excited states (where it is attractive) due to the existence of good diquarks with spin 0 in the wave function.

One may finally wonder if the Coulomb and linear confining strengths of the $3 Q$ potential that we have found reproducing the nonperturbative lattice QCD results of Refs. [4, 5] are unique. To answer this question we have considered the most relevant states, the first, $E_{2}\left(3 / 2^{+}\right)$, and second, $E_{3}\left(3 / 2^{+}\right)$, radial excitations and the first orbital excitation, $E_{1}\left(1 / 2^{-}\right)^{1}$. We have represented in Fig. 4 the sets of parameters that are able to simultaneously reproduce the positive and negative parity excited states for the $b b b$ system with a reasonable bottom quark mass, $m_{b}=4.655 \mathrm{GeV}$. As one can see there is a small range of parameters reproducing the general pattern of the $b b b$ spectrum. The strengths of the Coulomb and linear confining potentials we have determined giving rise to the results shown in Fig. 2 ( $A=0.1875$ and $\left.B=0.1374 \mathrm{GeV}^{2}\right)$, are in the middle of the aforementioned area where the most important states are properly reproduced. In Fig. 5 we have represented the parameters describing the $b b b$ and the $c c c$ systems with a simple Coulomb plus a linear confining potential, using a reasonable charm quark mass, $m_{c}=1.6 \mathrm{GeV}$. There are mainly two noticeable aspects. First of all, the smallness of the area reproducing the $c c c$ data. Even more, such area is in the limits of the uncertainties of the nonperturbative lattice QCD data for the $c c c$ system. The second relevant aspect is that there is no overlapping between the $c c c$ and $b b b$ range of parameters. As has been already noted, these results could indicate

\footnotetext{
${ }^{1}$ Having in mind the level degeneracies existing in the absence of rotational symmetry breaking, these states represent the majority of the spectra [4].
} 


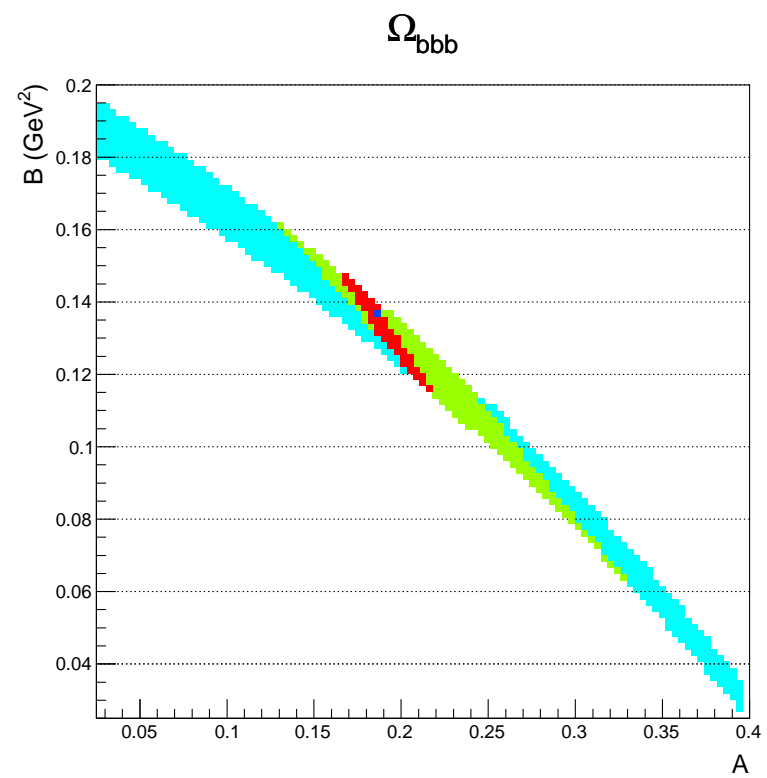

FIG. 4: Coulomb and linear confining strengths reproducing selected states of the $b b b$ system. The light blue area stands for sets of parameters reproducing the $E_{2}\left(3 / 2^{+}\right)$energy, the green area reproduces the $E_{2}\left(3 / 2^{+}\right)$and $E_{3}\left(3 / 2^{+}\right)$energies, the read surface reproduces the $E_{2}\left(3 / 2^{+}\right)$, $E_{3}\left(3 / 2^{+}\right)$, and $E_{1}\left(1 / 2^{-}\right)$energies. The dark blue point corresponds to our best fit.

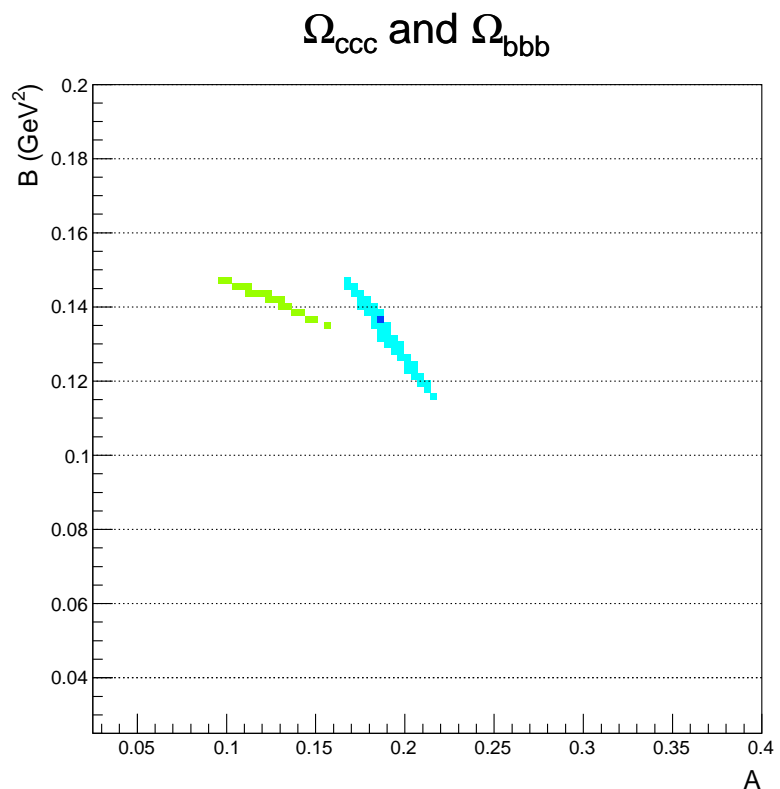

FIG. 5: The light blue area stands for Coulomb and linear confining strengths reproducing the $E_{2}\left(3 / 2^{+}\right), E_{3}\left(3 / 2^{+}\right)$, and $E_{1}\left(1 / 2^{-}\right)$energies of the $b b b$ system. The green surface represents the same for the $c c c$ system. The dark blue point corresponds to our best fit. 


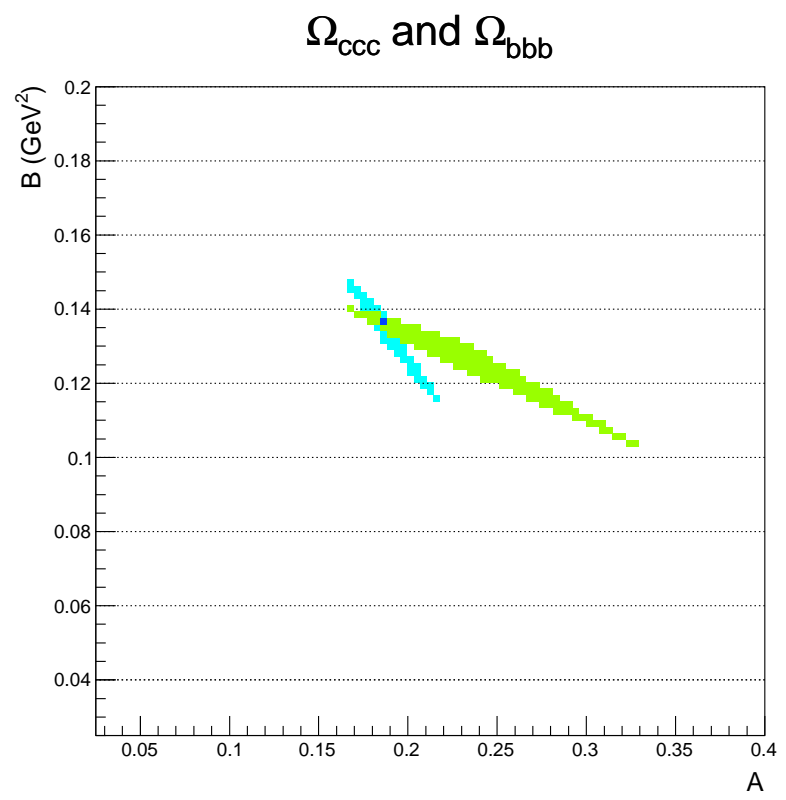

FIG. 6: The light blue area stands for Coulomb and linear confining strengths reproducing the $E_{2}\left(3 / 2^{+}\right), E_{3}\left(3 / 2^{+}\right)$, and $E_{1}\left(1 / 2^{-}\right)$energies of the $b b b$ system. The green surface represents the same for the $c c c$ system considering the spin-spin interaction of Eq. (3). The dark blue point corresponds to our best fit.

that the spectrum of the $c c c$ system hardly accommodates to a simple linear confining plus Coulomb potential, needing of higher order terms in the interaction. If we repeat the same simulation for the $c c c$ system adding the spin-spin force of Eq. (3), we get the results shown in Fig. 6, where one can see the overlapping between the $b b b$ and $c c c$ sets of parameters that coincides exactly in the Coulomb and linear strengths we have determined.

Let us finally comment on the possibility of applying the approach outlined in this work to light flavor sectors, as for example the excited spectrum of the $\Omega_{\text {sss }}$ baryon that has also been studied by means of lattice QCD [30]. Unfortunately the role played by other contributions beyond the confinement and the Coulomb potentials, as illustrated in Ref. [31], leads to the conclusion that only baryons made of heavy flavors are clean enough to properly disentangle confinement and Coulomb contributions.

\section{SUMMARY}

To summarize, the spectra of baryons containing three identical heavy quarks, $b$ or $c$, have been recently calculated in nonperturbative lattice QCD. The energy of the ground state and the lowest positive and negative parity excited states has been determined with high precision. These achievements constitute a unique opportunity to test phenomenological potential models in the regime in which they are expected to work best. We have analyzed these results by means of a Cornell-like potential using different numerical techniques for the three-body problem. For the case of the $b b b$ system a good agreement is obtained by means of a simple Coulomb plus linear confining potential. For the case of the $c c c$ system the additional contribution of the spin-spin interaction is needed to have a reliable mass 
for the charm quark. The spin-spin interaction comes suppressed by $M_{Q}^{-2}$, but it helps to correctly allocate the negative parity excitations with respect to the radial excitations of the $3 / 2^{+}$ground state. As in the case of the heavy meson spectra, a larger value of the Coulomb strength than predicted by $\mathrm{SU}(3)$ lattice QCD is needed. The phenomenological strengths of the Coulomb potential reproducing the heavy meson and the triply-heavy baryon spectra satisfy $A / a<1 / 2$, slightly different from the $1 / 2$ rule as the one-gluon exchange result. The strength of the linear confining interaction has to be also larger than derived from $\mathrm{SU}(3)$ lattice QCD. Our results support a coherent description of the $b b b$ and $c c c$ heavy-baryon spectra with the same Coulomb and confining strengths.

Let us conclude by emphasizing that the recent improvements in lattice QCD calculations of the heavy-baryon spectra [32] may benefit of constituent quark model predictions, and, on the other hand, potential model approaches do also require guidance of lattice data for constraining not only the parameter space but also the possible functional forms to be explored.

\section{Acknowledgments}

We thank to S. Meinel for valuable discussion and information about the present status of nonperturbative lattice QCD calculations of excited heavy baryon states. We also thank to N. Mathur for providing us with the numerical data of the triply charm baryon spectrum. This work has been partially funded by Ministerio de Educación y Ciencia and EU FEDER under Contract No. FPA2013-47443 and by the Spanish Consolider-Ingenio 2010 Program CPAN (CSD2007-00042) and by Generalitat Valenciana PrometeoII/2014/066. A.V. is thankful for financial support from the Programa Propio I of the University of Salamanca.

[1] J. Vijande, A. Valcarce, and H. Garcilazo, Phys. Rev. D 90, 094004 (2014).

[2] T. T. Takahashi, H. Suganuma, Y. Nemoto, and H. Matsufuru, Phys. Rev. D 65, 114509 (2002).

[3] S. Meinel, Phys. Rev. D 82, 114514 (2010).

[4] S. Meinel, Phys. Rev. D 85, 114510 (2012).

[5] M. Padmanath, R. G. Edwards, N. Mathur, and M. Peardon, Phys. Rev. D 90, 074504 (2014); PoS LATTICE2013, 247 (2014).

[6] C. Quigg and J. L. Rosner, Phys. Rep. 56, 167 (1979).

[7] E. Eichten, K. Gottfried, T. Kinoshita, K. D. Lane, and T. -M. Yan, Phys. Rev. D 21, 203 (1980).

[8] E. Eichten, S. Godfrey, H. Mahlke, and J. L. Rosner, Rev. Mod. Phys. 80, 1161 (2008).

[9] J. D. Bjorken, Report No. FERMILAB-Conf-85/69 (1985).

[10] Y. -Q. Chen and S. -Z. Wu, J. High Energy Phys. 08 (2011) 144; 09, 089(E) (2011).

[11] J. Vijande and A. Valcarce, Symmetry 1, 155 (2009).

[12] A. Valcarce, H. Garcilazo, and J. Vijande, Eur. Phys. J. A 37, 217 (2008).

[13] H. Garcilazo, A. Valcarce, and J. Vijande, J. Phys. G 34, 961 (2007).

[14] J. -M. Richard, arXiv:1205.4326. 
[15] E. Eichten, K. Gottfried, T. Kinoshita, J. Kogut, K. D. Lane, and T. M. Yan, Phys. Rev. Lett. 34, 369 (1975).

[16] G. S. Bali, Phys. Rep. 343, 1 (2001), and references therein.

[17] K. J. Juge, J. Kuti, and C. Morningstar, Phys. Rev. Lett. 90, 161601 (2003).

[18] P. Hasenfratz, R. R. Horgan, J. Kuti, and J. M. Richard, Phys. Lett. B 95, 299 (1980).

[19] T. T. Takahashi and H. Suganuma, Phys. Rev. D 70, 074506 (2004).

[20] V. G. Bornyakov, H. Ichie, Y. Mori, D. Pleiter, M. I. Polikarpov, G. Schierholz, T. Streuer, H. Stüben, and T. Suzuki (DIK collaboration), Phys. Rev. D 70, 054506 (2004).

[21] J. M. Cornwall, Phys. Rev. D 69, 065013 (2004).

[22] C. Alexandrou, Ph. de Forcrand, and O. Jahn, Nucl. Phys. B, Proc. Suppl. 119, 667 (2003).

[23] N. Isgur and G. Karl, Phys. Rev. D 18, 4187 (1978).

[24] M. Oka and K. Yazaki, Prog. Theor. Phys. 66, 556 (1981).

[25] B. Silvestre-Brac, Few-Body Syst. 20, 1 (1996).

[26] E. Klempt and J. -M. Richard, Rev. Mod. Phys. 82, 1095 (2010).

[27] V. Crede and W. Roberts, Rept. Prog. Phys. 76, 076301 (2013).

[28] N. Mathur (private communication).

[29] L. Liu, G. Moir, M. Peardon, S. M. Ryan, C. E. Thomas, P. Vilaseca, J. J. Dudek, R. G. Edwards, B. Joó, and D. G. Richards (Hadron Spectrum Collaboration), J. High Energy Phys. 07 (2012) 126.

[30] J. Bulava, R. G. Edwards, E. Engelson, B. Joó, H. -W. Lin, C. Morningstar, D. G. Richards, and S. J. Wallace, Phys. Rev. D 82, 014507 (2010).

[31] A. Valcarce, H. Garcilazo, and J. Vijande, Phys. Rev. C 72, 025206 (2005).

[32] Z. S. Brown, W. Detmold, S. Meinel, and K. Orginos, Phys. Rev. D 90, 094507 (2014). 